Pacific Journal of Mathematic 


\title{
AN ALGEBRAIC CHARACTERIZATION OF FIXED IDEALS \\ IN CERTAIN FUNCTION RINGS
}

\author{
Lyle E. Pursell
}

1. Introduction. In this paper an algebraic characterization of the fixed ideals in a certain class of function rings is given (an ideal in a function ring is fixed if there is a point at which all functions in the ideal vanish). This class of function rings includes the rings of all real-, complex-, or quaternion-valued continuous functions on a normal Hausdorff space whose points are $G$-delta sets and the ring of $r$-fold differentiable functions on an $r$-differentiable manifold whose coordinate covering is neighborhood finite. For these rings of functions we construct the underlying space from the fixed ideals in the same way that Gelfand and Kolmogoroff [3] have constructed a compact space from the non-unit ideals in its ring of all real-valued continuous functions.

We also show the existence of certain homomorphisms from the automorphism groups of these function rings into the group of homeomorphisms of the underlying space onto itself. In $\S 5$ we find that an isomorphism between the rings of all $r$-differentiable functions on two $r$-differentiable manifolds can be extended to an isomorphism between the rings of all continuous functions on these manifolds and that the homeomorphism determined by this isomorphism is differentiable.

\section{The general case.}

(2.1) By $\mathscr{R}$ we mean a ring of functions from a regular Hausdorff space $X$ to a division ring $D$ having the following properties:

$P_{1}$. If $f$ is in $\mathscr{C}_{2}$, then the set of zeros of $f$, which we denote by $Z(f)$, is closed.

$P_{2}$. If $x$ is not in a closed set $F$, then there is a function $f$ in $\mathscr{R}$ such that $Z(f)$ contains a neighborhood of $F$ but does not contain $x$.

$P_{3}$. If $f$ in $\mathscr{R}$ does not vanish at any point of a closed set $F$, then there is a function $g$ in $\mathscr{R}$ such that $f g$ (and also $g f$ ) has the value 1 at every point of $F$.

$P_{4}$. For each $x$ in $X$ there is a function $f_{x}$ in $\mathscr{T H}$ which vanishes at $x$ and only at $x$.

Received June 14, 1954. This paper includes extensions of certain results given in the author's doctoral thesis which was written at Purdue University under the direction of Prof. M. E. Shanks (1952). The author wishes to express his appreciation to Prof. Shanks, Prof. M. Henriksen, and Dr. D. W. Dubois, who made several contributions to this paper, 
(2.2) By the "support of a function $f$ " in . $\mathrm{Sp}(f)$, we mean the set $\mathrm{Cl}(X-Z(f))$, From the properties of closure we have:

(i) $\operatorname{Sp}(f)=X-\operatorname{Int} Z(f)$,

(ii) $\operatorname{Int} \operatorname{Sp}(f)=X-\mathrm{Cl} \operatorname{Int} Z(f)$,

(iii) $\operatorname{Sp}(f)=\mathrm{Cl} \operatorname{Int} \mathrm{Sp}(f)$.

By the "annihilator of a function $f$ " in $\mathscr{R}$, which we denote by $A(f)$, we mean the set of all $g$ in $\mathscr{R}$ such, that $f g=0$ (and hence $g f=$ $0)$. For any ring of functions with values in a division ring the annihilator of an element is a two-sided ideal. In addition we have

$$
A(f)=\{g \in \mathscr{R} \mid Z(g) \supset \operatorname{Sp}(f)\},
$$

and $A(f)=\mathscr{R}$ if an only if $f=0$.

(2.3) Lemma. If $f$ and $g$ are in $\mathscr{R}$ and $g \neq 0$, then $Z(f)$ and $\mathrm{Sp}(g)$ are disjoint if and only if $f-A(g)$ has an inverse in the residue class ring $\mathscr{R}-A(g)$.

Proof. Since $g \neq 0$, then $Z(g) \neq X$ and $\operatorname{Sp}(g)$ is not empty. If $f$ does not vanish at any point of $\operatorname{Sp}(g)$, then there is a function $h$ in $\mathscr{R}$ such that $f h$ and $h f$ have the value 1 at every point of $\operatorname{Sp}(g)$, that is, $f h \equiv 1(\bmod A(g))$ and $h f \equiv 1(\bmod A(g))$. Hence $f-A(g)$ has an inverse in $\mathscr{R}-A(g)$. If $f-A(g)$ has an inverse in $\mathscr{R}-A(g)$, then there is a function $h$ in $\mathscr{R}$ such that $(f h-1)$ is in $A(g)$, that is $(f h-1)$ vanishes at every point of $\operatorname{Sp}(g)$. Hence $f$ does not vanish at any point of $\operatorname{Sp}(g)$.

(2.4) If $f$ is in $\mathscr{R}$, let $H(f)$ be the set of all nonzero $g$ in $\mathscr{R}$ such that $f-A(g)$ has an inverse in the ring $\mathscr{R}-A(g)$. An ideal I in $\mathscr{R}$ is "bounded" if there is a function $f$ in . that $H(f)$ contains $H(g)$ for every $g$ in $I$. We say that " $I$ is bounded by $f$ ". An ideal which is maximal in the set of all bounded ideals is called a "maximal bounded ideal". The set of all maximal bounded ideals is denoted by $M[\mathscr{R}]$. We observe that an ideal contained in a bounded ideal is bounded.

(2.5) Lemma. For $f$ and $g$ in $\mathscr{R}, Z(g)$ contains $Z(f)$ if and only if $H(f)$ contains $H(g)$.

Proof. From (2.3) and (2.4) $H(f)$ is the set of all functions $h$ in $\mathscr{R}$ such that $Z(f)$ and $\operatorname{Sp}(h)$ are disjoint. Hence if $Z(f)$ is a subset of $Z(g)$, then $H(f)$ contains $H(g)$. Suppose there is a point $x$ in $Z(f)$ 
but not in $Z(g)$, then by $P_{2}$ there is a function $h$ in $\mathscr{R}$ which is different from zero on a neighborhood of $x$ but vanishes on a neighborhood of $Z(g)$. For this function $h, Z(f)$ meets $\operatorname{Sp}(h)$ but $Z(g)$ does not. Hence if $Z(f)$ is not a subset of $Z(g)$, then $H(f)$ does not contain $H(g)$.

(2.6) THEOREM. If $\mathscr{R}$ is a ring of functions from a regular. Hausdorff space $X$ to a division ring $D$ which satisfies $P_{1}, P_{2}, P_{3}$, and $P_{4}$ of (2.1), then an ideal $I$ in $\mathscr{R}$ is a fixed ideal if and only if it is a bounded ideal.

Proof. If $I$ is a fixed ideal, then there is a point $x$ at which all elements of $I$ vanish. From $P_{4}$ there is a function $f_{x}$ in $\mathscr{R}$ which vanishes at $x$ and only at $x$. For every $g$ in $I, Z(g)$ contains $Z\left(f_{x}\right)$, that is $H\left(f_{x}\right)$ contains $H(g)$. Since $f_{x}$ has no inverse, $I$ is bounded. If $I$ is bounded by a function $f$ in $\mathscr{R}$ without an inverse, then $Z(f)$ is a subset of $Z(g)$ for every $g$ in $I$. Since $Z(f)$ is not empty, $I$ is fixed.

(2.7) For $x$ in $X, I(x)$ means the fixed ideal $\{f \in \mathscr{R} \mid f(x)=0\}$. From (2.6) an ideal is a maximal bounded ideal if and only if it is of this form.

\section{Let $A$ be a subset of $M[\mathscr{R}]$. If we define}

$$
J \in \mathrm{Cl}(A) \text { if and only if } J \supset \bigcap_{I \in A} I
$$

for $A$ nonempty and $\mathrm{Cl}(A)=A$ for $A$ empty, then $M[\mathscr{R}]$ is said to have the "Stone topology". We denote the set $M[\mathscr{R}]$ with the Stone topology by $X^{*}$.

(2.9) TheOREM. If $\mathscr{R}$ is a ring of functions from a regular Hausdorff space $X$ to a division ring $D$ satisfying $P_{1}, P_{2}, P_{3}$, and $P_{4}$ of (2.1), then $X$ is homeomorphic to $X^{*}$.

Proof. From (2.7) the mapping $x \rightarrow I(x)$ is a one-to-one mapping of $X$ onto $X^{*}$. Let $a \in \mathrm{Cl}(A), A \subset X$, and let $A^{*}$ be the image of $A$ under the mapping $x \rightarrow I(x)$, then every function in $\mathscr{R}$ vanishing on $A$ (that is, every function in $\left.\bigcap_{X \in A} I(x)=\bigcap_{I \in A^{*}} I\right)$ also vanishes at $a$ (that is, is in $I(a)$ ) and $I(a)$ is in $\mathrm{Cl}\left(A^{*}\right)$. If, however, $a$ is not in $\mathrm{Cl}(A)$, then there is a function $f$ in $\mathscr{R}$ vanishing on $A$ but not at $a$. Then $f$ is in $\bigcap_{I \in A^{*}} I$ but not in $I(\alpha)$, and $I(\alpha)$ is not in $\mathrm{Cl}\left(A^{*}\right)$. Hence the correspondence $x \rightarrow I(x)$ is a homeomorphism of $X$ onto $X^{*}$.

(2.10) CoROllary. If the rings $\Re$ and $\Re^{\prime}$ of functions from the regular Hausdorff spaces $X$ and $X^{\prime}$ to the division rings $D$ and $D^{\prime}$, 
respectively, satisfy $P_{1}, P_{2}, P_{3}$, and $P_{i}$ of (2.1) and are isomorphic, then the spaces $X$ and $X^{\prime}$ are homeomorphic.

(2.11) Let $i: \quad h \rightarrow \aleph^{\prime}$ be the isomorphism referred to in the preceding paragraph. With the point $x$ in $X$ we associate the $x^{\prime}$ (which exists uniquely) in $X^{\prime}$ which is the common zero of all functions in the isomorphic image of the ideal consisting of all functions in which vanish at $x$, that is,

$$
x^{\prime}=\bigcap_{f \in i(I(x))} Z(f) .
$$

One can show that the correspondence $x \rightarrow x^{\prime}$ is a homeomorphism from $X$ onto $X^{\prime}$. We will denote this homeomorphism by $\phi(i)$ and refer to it as " the homeomorphism from $X$ onto $X$ ' corresponding to (or determined by) the isomorphism i from " $X^{\prime \prime}$ onto

(2.12) By . ( $(2)$ we mean the automorphism group of . By $\mathscr{H}(X)$ we mean the homeomorphism group of $X$, that is the group of all homeomorphisms of $X$ onto itself. If $i_{1}$ and $i_{2}$ are in $/\left(i_{1}\right)$, then it follows from (2.11) that $\phi\left(i_{1} i_{2}\right)=\phi\left(i_{1}\right) \phi\left(i_{2}\right)$. Hence we have the theorem of the following paragraph.

(2.13) TheOREM. The mapping $\phi: \quad \mathscr{Z}(\mathscr{R}) \rightarrow \mathscr{H}(X)$ is a homomorphism from the automorphism group of into the homeomorphism group of $X$.

(2.14) For $x$ in $X$ we denote the set of values $\{f(x) \mid f \in \Re\}$ by $V(x)$. From $P_{2}$ and $P_{3}$ the set $V(x)$ is a subdivision ring of $D$. The correspondence $f \rightarrow f(x)$ is a homomorphism from onto $V(x)$ with kernel $I(x)$, hence the correspondence $f-I(x) \rightarrow f(x)$ is an isomorphism from the residue class ring $\mathscr{R}-I(x)$ onto $V(x)$. Since $\mathscr{R}-I(x)$ is, therefore, a division ring, $I(x)$ is a maximal ideal, that is, every maximal bounded ideal is a maximal ideal.

(2.15) Lemma. If $f \rightarrow f^{\prime}$ is an isomorphism from onto $r^{\prime}$ and $x \rightarrow x^{\prime}$ is the corresponding homeomorphism, then the correspondence $f(x) \rightarrow f^{\prime}\left(x^{\prime}\right)$ is an isomorphism from $V(x)$ onto $V^{\prime}\left(x^{\prime}\right)$.

Proof. Since $I^{\prime}\left(x^{\prime}\right)$ is the isomorphic image of $I(x)$, the correspondence $f-I(x) \rightarrow f^{\prime}-I^{\prime}\left(x^{\prime}\right)$ is an isomorphism. Since $f(x) \rightarrow f-I(x)$ and $f^{\prime}-I^{\prime}\left(x^{\prime}\right) \rightarrow f^{\prime}\left(x^{\prime}\right)$ are isomorphisms, $f(x) \rightarrow f^{\prime}\left(x^{\prime}\right)$ is an isomorphism from $V(x)$ onto $V^{\prime}\left(x^{\prime}\right)$.

\section{Rings of continuous functions.}

(3.1) Čech [2] has shown that a subset of a normal Hausdorff 
space is the zero set of some real-valued continuous function if and only if it is a closed $G_{\delta}$ set. Using his result and Urysohn's lemma concerning real-valued continuous functions on a normal space, one may show that the rings $-C(X, R)$ of all real-valued continuous functions on $X, C(X, K)$ of all complex-valued continuous functions on $X$, and $C(X, Q)$ of all quaternion-valued continuous functions on $X$-satisfy $P_{1}$, $P_{2}, P_{3}$, and $P_{4}$ of (2.1) if $X$ is a normal Hausdorff space all of whose points are $G_{\delta}$ sets. Hence we have the following.

(3.2) Theorem. Let $X$ and $X^{\prime}$ be normal Hausdorff spaces all of whose points are $G_{\delta}$ sets and let $F$ denote either the real field, the complex field, or the quaternion ring. If $C(X, F)$ and $C\left(X^{\prime}, F\right)$ are isomorphic, then $X$ and $X^{\prime}$ are homeomorphic.

(3.3) According to results obtained by Gelfand and Kolmogoroff [3], Hewitt [6], and Gillman, Henriksen, and Jerison [4], Theorem (3.2) holds for completely regular spaces satisfying the first axiom of countability. There are, however, normal spaces all of whose points are $G_{\delta}$ sets which do not satisfy the first axiom of countability (cf. Bing [1, p. 180, Example C]).

(3.4) For the rings $C(X, F)$ it can be established that the homomorphism $\phi: \mathscr{A}(C(X, F)) \rightarrow \mathscr{H}(X)$ of (2.11) and (2.13) is a homomorphism onto $\mathscr{H}(X)$.

\section{Rings of real-valued functions.}

(4.1) If $\mathscr{R}$ is a ring of real-valued functions on $X$ satisfying $P_{1}$, $P_{2}, P_{3}$, and $P_{4}$, then for each $x$ the set of values $V(x)=\{f(x) \mid f \in R\}$ is a subfield of the real field $R$. We now introduce an additional property for the ring $\mathscr{R}$ :

$P_{5}$. For each $x$ in $X$ the set of values $V(x)$ is a subfield of the real field $R$ which has only one isomorphism into $R$, the identity isomorphism.

Property $P_{5}$ holds if $V(x)=R$; hence $C(X, R)$ satisfies $P_{5}$. There are rings of real-valued functions satisfying $P_{1}, P_{2}, P_{3}, P_{4}$, and $P_{5}$ which contain discontinuous functions as is shown by the example of the following paragraph given to the author by D. W. Dubois.

(4.2) Example. Let $X$ be the closed interval $[0,1], \alpha$ be a finite subdivision $\left\{0=x_{0}, x_{1}, \cdots, x_{n-1}, x_{n}=1\right\}$ of $X$, and $A$ be the set of all $\alpha$. Let $\theta(x)=\exp (x)$ for $x \neq 0$ and $\theta(0)=0$. Let $B(\alpha)$ be the set of all real-valued functions $f$ on $X$ such that 


$$
f(x)=\begin{aligned}
& p_{i}(x, \theta(x)) \\
& q_{i}(x, \theta(x))
\end{aligned},
$$$$
x_{i-1} \leq x \leq x_{i}, i=1,2, \cdots, n \text {, }
$$

where $p_{i}(x, \theta)$ and $q_{i}(x, \theta)$ are polynomials in $x$ and $\theta(x)$ such that $f(x)$ is continuous at $x_{1}, x_{2}, \cdots, x_{n-2}$, and $x_{n-1}$ and $q_{i}(x, \theta(x))$ does not vanish for $x_{i-1} \leq x \leq x_{i}$ for any $i$. If $h=\bigcup_{\alpha \in 1} B(\alpha)$, then is a ring of real-valued functions which satisfy $P_{1}, P_{2}, P_{3}, P_{4}$, and $P_{5}$ but some of which are discontinuous.

(4.3) Theorem (4.4) and (4.5) may be established by using $P_{5}$ and the results of $\S 2$.

(4.4) THEOREM. If $\bar{R}$ and are isomorphic rings of real valued functions on regular Hausdorff spaces $X$ and $X^{\prime}$ satisfying $P_{1}, P_{2}$, $P_{3}, P_{4}$, and $P_{5}, i$ is the isomorphism from $\mathscr{R}$ onto $\mathscr{R}^{\prime}$, and $h$ is the corresponding homeomorphism from $X$ onto $X^{\prime}$, then:

(i) $f(x)=(i f)(h(x))$ for all $f$ in $R$ and $x$ in $X$. Hence $f$ is bounded above (below) if and only if (if) is bounded above (below); lub $f=\operatorname{lub} f^{\prime}$, glb $f=\mathrm{glb} f^{\prime}$; and the subrings of all bounded functions in $R$ and $R^{\prime}$ are isomorphic.

(ii) There is an isomorphism $i^{*}$ from $C(X, R)$ onto $C\left(X^{\prime}, R\right)$ such that $i(f)=i^{*}(f)$ for all $f$ in $C(X, R) \cap \mathscr{R}$.

(4.5) THEOREM. If $\mathscr{R}$ is a ring of real-valued functions on a regular Hausdorff space satisfying $P_{1}, P_{2}, P_{3}, P_{4}$, and $P_{5}$, then the homomorphism $\phi$ of (2.11) and (2.13) is an isomorphism of $\mathscr{Y}(R)$ into $\mathscr{H}(X)$.

From (3.4) and (4.5) we have the following.

(4.6) Theorem. The groups $\mathscr{Z}(C(X, R))$ and $\mathscr{H}(X)$ are isomorphic.

\section{Rings of continuously differentiable functions.}

(5.1) If $C^{r}(M)$ is the ring of $r$-fold continuously differentiable functions on an $r$-differentiable manifold $M$ with a neighborhood-finite covering of coordinate neighborhoods $(r$ may be either a positive integer or the symbol $\infty$ ), then $C^{r}(M)$ satisfies $P_{1}, P_{2}, P_{3}, P_{4}$, and $P_{5}$. The theorem of the following paragraph may be obtained.

(5.2) Theorem. If $C^{r}(M)$ and $C^{r^{\prime}}\left(M^{\prime}\right)$ are isomorphic, then $M$ and $M^{\prime}$ are homeomorphic. The homeomorphism $h$ determined by the isomorphism is differentiable (that is, $f(h)$ is in $C^{r}(M)$ if $f$ is in $C^{r^{\prime}}\left(M^{\prime}\right)$ ) and the isomorphism can be extended to an isomorphism from $C(M, R)$ onto $C\left(M^{\prime}, R\right)$. 
6. Additional remarks. Since the above was written the author has observed that $P_{4}$ may be replaced by the weaker hypothesis:

$P_{4}$. For each $x$ in $X$ there is a pair of functions $g$ and $h$ in.$h$ such that $x=Z(g)-Z(h)$.

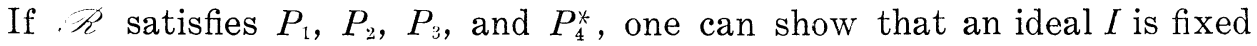
if and only if there is a pair of functions $g$ and $h$ in $R$ such that $H(g)$ does not contain $H(h)$ but $H(g h)$ does contain $H(f h)$ for every $f$ in the ideal $I$. (Lemma (2.5) holds as before.) The results of (2.9) through (2.15) may then be established if $X^{*}$ is defined to be the set of maximal fixed ideals with the Stone topology.

If $X$ is a completely regular, locally-compact space all of whose points are $G_{\delta}$ sets, then the rings $C_{0}(X, R), C_{0}(X, K)$, and $C_{0}(X, Q)$ of all real-, complex-, or quaternion-valued continuous functions with compact supports satisfy $P_{1}, P_{2}, P_{3}$, and $P_{1}^{*}$. Hence it follows that they determine $X$. (This result for $C_{0}(X, R)$ has already been established by Shanks [7] without assuming that points are $G_{\delta}$ sets). One can also show that the automorphism group $C^{\prime}\left(C_{0}(X, R)\right)$ is isomorphic to $\mathscr{L}(C(X, R))$ and $\mathscr{L}(X)$.

\section{REFERENCES}

1. R. H. Bing, Metrization of topological spaces, Canadian J. Math., 3 (1951), 175-186.

2. E. Čech, On bicompact spaces, Ann. Math., 38 (1937), 823-844.

3. I. Gelfand and A. N. Kolmogoroff, On rings of continuous functions on topological spaces, C. R. (Doklady) Acad. Sci. URSS, 22 (1939), 11-15.

4. L. Gillman, M. Henriksen, and M. Jerison, A theorem of Gelfand and Kolmogoroff concerning maximal ideals in rings of continuous functions, Proc. Amer. Math. Soc., 5 (1954), 447-455.

5. M. Hall, Jr., A type of algebraic closure, Ann. Math., 40 (1939), 360-369.

6. E. Hewitt, Rings of real-valued continuous functions I, Trans. Amer. Math. Soc., 64 (1948), 45-99.

7. M. E. Shanks, Rings of functions on locally compact spaces, (abstract no. 365), Bull. Amer. Math. Soc., 57 (1951), 295.

8. N. Steenrod, The topology of fibre bundles, Princeton University Press, 1951.

9. M. H. Stone, Applications of the theory of Boolean rings to general topology, Trans. Amer. Math. Soc., 41 (1937), 375-481.

GrinNell COLLEge 

.././. ./FrontMatter/paper .pdf 


\section{Pacific Journal of Mathematics}

Nesmith Cornett Ankeny and Theodore Joseph Rivlin, On a theorem of S.

Bernstei........................................ 849

Louis Auslander, The use of forms in variational calculation .......... 853

Paul Civin, Abstract Riemann sum . .......................... 861

Paul Civin, Some ergodic theorems involving two operator ............ 869

Eckford Cohen, The number of solutions of certain cubic congruence .... . 877

Richard M. Cohn, Specializations over difference field .............. 887

Jean Dieudonné, Pseudo-discriminant and Dickson invarian . . ......... 907

Ky Fan, A comparison theorem for eigenvalues of normal matrice ........ 911

Richard P. Gosselin, On the convergence behaviour of trigonometric interpolating polynomial ........................... 915

Peter K. Henrici, On generating functions of the Jacobi polynomial . . . . . . . 923

Meyer Jerison, An algebra associated with a compact grou ............ 933

Wilhelm Magnus, Infinite determinants associated with Hill's equatio . . . . . 941

G. Power and D. L. Scott-Hutton, The slow steady motion of liquid past a semi-elliptical bos................................. 953

Lyle E. Pursell, An algebraic characterization of fixed ideals in certain function ring .................................... 963

C. T. Rajagopal, Additional note on some Tauberian theorems of O. Szás . . 971 Louis Baker Rall, Error bounds for iterative solutions of Fredholm integral

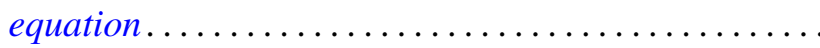

Shigeo Sasaki and Kentaro Yano, Pseudo-analytic vectors on

pseudo-Kählerian manifold ......................

Eugene Schenkman, On the tower theorem for finite group

P. Stein and John E. L. Peck, On the numerical solution of Poisson's equation over a rectangl ........................

Morgan Ward, The mappings of the positive integers into themselves which preserve divisio .

Seth Warner, Weak locally multiplicatively-convex algebra 1025

Louis Weisner, Group-theoretic origin of certain generating function .... 